\title{
The Beta Log-logistic Weibull Distribution: Model, Properties and Application
}

\author{
Boikanyo Makubate $^{1}$, Broderick O. Oluyede ${ }^{2}$, Neo Dingalo ${ }^{1}$ \& Adeniyi Francis Fagbamigbe ${ }^{1,3}$ \\ ${ }^{1}$ Department of Mathematics and Statistical Sciences, Botswana International University of Science and Technology, \\ Palapye, BW \\ ${ }^{2}$ Department of Mathematical Sciences, Georgia Southern University, Statesboro, GA, 30460, USA \\ ${ }^{3}$ Department of Epidemiology and Medical Statistics, University of Ibadan, Nigeria \\ Correspondence: B. O. Oluyede, Department of Mathematical Sciences, Georgia Southern University, Statesboro, GA, \\ 30460, USA.
}

Received: August 25, 2018 Accepted: September 11, 2018 Online Published: September 12, 2018

doi:10.5539/ijsp.v7n6p49 URL: https://doi.org/10.5539/ijsp.v7n6p49

\begin{abstract}
We propose and develop the properties of a new generalized distribution called the beta log-logistic Weibull (BLLoGW) distribution. This model contain several new distributions such as beta log-logistic Rayleigh, beta log-logistic exponential, exponentiated log-logistic Weibull, exponentiated log-logistic Rayleigh, exponentiated log-logistic exponential, log-logistic Weibull, log-logistic Rayleigh and log-logistic distributions as special cases. Structural properties of this generalized distribution including series expansion of the probability density function and cumulative distribution function, hazard function, reverse hazard function, quantile function, moments, conditional moments, mean deviations, Bonferroni and Lorenz curves, Rényi entropy and distribution of order statistics are presented. The parameters of the distribution are estimated using maximum likelihood estimation technique. A Monte Carlo simulation study is conducted to examine the bias and mean square error of the maximum likelihood estimates. A real dataset is used to illustrate the applicability and usefulness of the new generalized distribution.
\end{abstract}

Keywords: generalized distributions, beta distribution, Weibull Distribution, maximum likelihood estimation

\section{Introduction}

Generalized distributions are of tremendous practical importance and have recieved considerable attention by many authors in recent years. Eugene, Lee and Famoye (2002) introduced a generalized beta distributions and presented results on the beta normal distribution. The work presented by Jones (2004) is also related to the beta-generated family. Recently, Barreto-Souza, Santos and Cordeiro (2010) introduced the beta generalized exponential distribution where they derived mathematical properties of the distribution. Also, Pescim et al. (2010) studied the beta generalized half-normal distribution. Nadarajah, Cordeiro \& Ortega (2012) presented general results on the beta modified Weibull distribution. Famoye, Lee \& Olumolade (2005) studied beta Weibull distribution and showed that the lifetime distribution is unimodal and Cordeiro and Nadarajah (2011) extended the beta Weibull distribution by beta exponentiated Weibull distribution. There are several generalizations of the log-logistic distribution including beta log-logistic distribution, presented by Lemonte (2014) and the log-logistic Weibull distribution by Oluyede et al. (2016).

The primary motivation for the development of the beta log-logistic Weibull distribution is the modeling of lifetime data and other data types with a diverse model that takes into consideration not only shape, and scale but also skewness, kurtosis and tail variation. Also, motivated by various applications of log-logistic, Weibull and beta distributions in several areas including reliability, exponential tilting (weighting) in finance and actuarial sciences, as well as economics, where loglogistic distribution plays an important role in income, we construct and develop the statistical properties of this new class of generalized distribution called the beta log-logistic Weibull distribution and apply it to real lifetime data in order to demonstrate the usefulness of the proposed distribution.

In this paper, the results are organized in the following manner. The beta log-logistic Weibull (BLLoGW) distribution, its sub-models, quantile function, hazard and reverse hazard functions are given in section 2 . In section 3 , moments, moment generating function and conditional moments are presented. Mean deviations, Lorenz and Bonferroni curves are given in section 4. Section 5 contain results on Rényi entropy, density of the order statistics and L-moments. Maximum likelihood estimates of the model parameters are given in section 6. A Monte Carlo simulation study to examine the bias and mean square error of the maximum likelihood estimates are presented in section 7 . Section 8 contains an application of the new model to real data set. A short conclusion is given in section 9 . 


\section{The Model Definition}

If $G(x)$ denote the cumulative distribution function of a random variable $X$, then a generalized class of distribution can be defined by

$$
F(x)=I_{G(x)}(a, b)=\frac{1}{B(a, b)} \int_{0}^{G(x)} t^{a-1}(1-t)^{b-1} d t,
$$

where the parameters $a$ and $b$ are shape parameters, $B(a, b)=\Gamma(a) \Gamma(b) / \Gamma(a+b)$ denote the beta function and $\Gamma($.$) is the$ gamma function. The corresponding probability density function (pdf) is given by

$$
f(x)=\frac{1}{B(a, b)}[G(x)]^{a-1}[1-G(x)]^{b-1} g(x),
$$

where $g(x)$ is the pdf of the baseline cumulative distribution function (cdf) $G(x)$.

\subsection{Beta Log-Logistic Weibull Distribution}

In this subsection, the model is presented. A series expansion of the BLLoGW cdf is given. The new BLLoGW cdf is given by

$$
\begin{aligned}
F_{\text {BLLoGW }}(x ; c, a, b, \alpha, \beta) & =\frac{1}{B(a, b)} \int_{0}^{G_{L L O G W}(x ; c, \alpha, \beta)} t^{a-1}(1-t)^{b-1} d t \\
& =I_{G_{L L o G W}(x ; c, \alpha, \beta)}(a, b),
\end{aligned}
$$

where $G_{\text {LLoGW }}(x ; c, \alpha, \beta)=1-\left(1+x^{c}\right)^{-1} e^{-\alpha x^{\beta}}$ is the log-logistic Weibull cdf (Oluyede et al., 2016) with parameters c, $\alpha$ and $\beta>0$. If $|t|<1$ and $b>0$ is real non-integer, we apply series representation

$$
(1-t)^{b-1}=\sum_{j=0}^{\infty} \frac{(-1)^{j} \Gamma(b)}{\Gamma(b-j) j !} t^{j}
$$

to the BLLoGW cdf to obtain

$$
\begin{aligned}
F_{\text {BLLOGW }}(x ; c, a, b, \alpha, \beta) & =\frac{1}{B(a, b)} \sum_{j=0}^{\infty} \frac{(-1)^{j} \Gamma(b)}{\Gamma(b-j) j !} \int_{0}^{\left[1-\left(1+x^{c}\right)^{-1} e^{-\alpha x^{\beta}}\right]} t^{a+j-1} d t \\
& =\frac{1}{B(a, b)} \sum_{j=0}^{\infty} \frac{(-1)^{j} \Gamma(b)\left[1-\left(1+x^{c}\right)^{-1} e^{-\alpha x^{\beta}}\right]^{a+j}}{\Gamma(b-j) j !(a+j)} \\
& =\sum_{j=0}^{\infty} w(j, a, b) G_{E L L O G W}(x),
\end{aligned}
$$

for $x>0, c>0, a>0, b>0, \alpha>0, \beta>0$, where $G_{E L L O G W}(x)$ is the exponentiated log-logistic Weibull cdf with the exponentiated parameter $a+j>0$. The corresponding pdf from equation (2) is given by

$$
\begin{aligned}
f_{B L L O W}(x ; c, a, b, \alpha, \beta) & =\frac{1}{B(a, b)}\left[1-\left(1+x^{c}\right)^{-1} e^{-\alpha x^{\beta}}\right]^{a-1}\left[\left(1+x^{c}\right)^{-1} e^{-\alpha x^{\beta}}\right]^{b} \\
& \times\left[c x^{c-1}\left(1+x^{c}\right)^{-1}+\alpha \beta x^{\beta-1}\right]
\end{aligned}
$$

for $x>0, c>0, a>0, b>0, \alpha>0, \beta>0$.

\subsection{Expansion of Density Function}

In this section, the expansion of the BLLoGW pdf is presented. Applying equation (4) to the expression

$$
\left(1-\left(1+x^{c}\right)^{-1} e^{-\alpha x^{\beta}}\right)^{a-1}=\sum_{i=0}^{\infty} \frac{(-1)^{i} \Gamma(a)}{i ! \Gamma(a-i)}\left(1+x^{c}\right)^{-i} e^{-\alpha(i) x^{\beta}},
$$

the BLLoGW pdf can be written as

$$
f_{B L L O G W}(x ; c, a, b, \alpha, \beta)=\frac{1}{B(a, b)} \sum_{i=0}^{\infty} \frac{(-1)^{i} \Gamma(a)}{i ! \Gamma(a-i)}\left(1+x^{c}\right)^{-(i+b)} e^{-\alpha(i+b) x^{\beta}}\left[c x^{c-1}\left(1+x^{c}\right)^{-1}+\alpha \beta x^{\beta-1}\right],
$$


for $x>0, c>0, a>0, b>0, \alpha>0, \beta>0$, where parameter $\alpha$ control the scale of the distribution and the parameters $c$, $\beta, a$, and $b$ control the shape, skewness and tail weight of the distribution.

Consequently, the BLLoGW pdf can be written as

$$
f_{B L L O W}(x ; c, a, b, \alpha, \beta)=\sum_{i=0}^{\infty} w(i, a, b) g(x ; c,(i+b), \alpha(i+b), \beta),
$$

where

$$
w(i, a, b)=\frac{(-1)^{i} \Gamma(a)}{i ! \Gamma(a-i) B(a, b)(i+b)}
$$

and $g(x ; c,(i+b), \alpha(i+b), \beta)$ is the pdf of the Burr XII Weibull distribution with parameters $c,(i+b), \alpha(i+b), \beta>0$. Plots of the BLLoGW pdf for selected values of the model parameters are given in Figure 1. The graphs of the BLLoGW

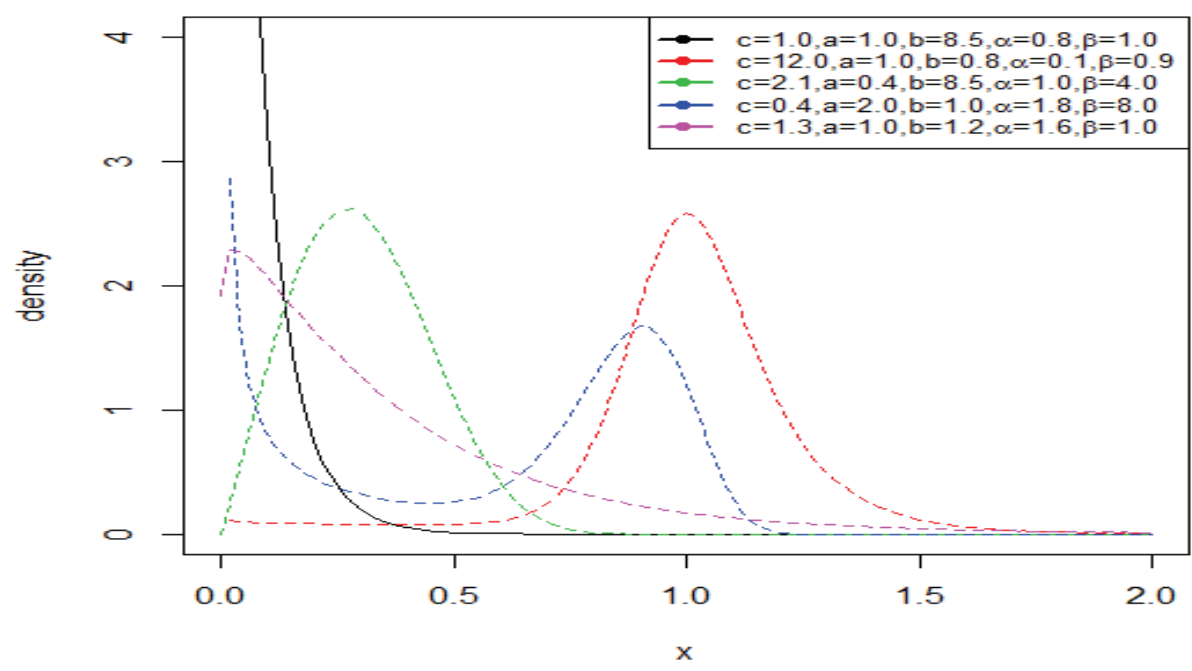

Figure 1. Plots of the BLLoGW Density Function

distribution can be increasing and decreasing L-shaped, uni-modal and decreasing-increasing-decreasing among many potential shapes. The survival or reliability function of the BLLoGW distribution is given by

$$
\bar{F}_{B L L G W}(x ; c, a, b, \alpha, \beta)=1-I_{G_{L L O G W}(x ; c, \alpha, \beta)}(a, b) .
$$

\subsection{Hazard and Reverse Hazard Functions}

The hazard and reverse hazard functions of the BLLoGW distribution are presented in this subsection. The hazard rate and reverse hazard functions of the BLLoGW distribution are given by

$$
h_{F}(x)=\frac{f_{B L L O G W}(x)}{\bar{F}_{B L L O G W}(x)}=\frac{\left[1-\left(1+x^{c}\right)^{-1} e^{-\alpha x^{\beta}}\right]^{a-1}\left[\left(1+x^{c}\right)^{-1} e^{-\alpha x^{\beta}}\right]^{b}}{B(a, b)\left(1-I_{\left[1-\left(1+x^{c}\right)^{-1} e^{-\alpha \beta^{\beta}}\right]}(a, b)\right)}\left[c x^{c-1}\left(1+x^{c}\right)^{-1}+\alpha \beta x^{\beta-1}\right] \text {, }
$$

and

$$
\tau_{F}(x)=\frac{f_{B L L o G W}(x)}{F_{B L L o G W}(x)}=\frac{\left[1-\left(1+x^{c}\right)^{-1} e^{-\alpha x^{\beta}}\right]^{a-1}\left[\left(1+x^{c}\right)^{-1} e^{-\alpha x^{\beta}}\right]^{b}}{B(a, b) I_{\left[1-\left(1+x^{c}\right)^{-1} e^{-\alpha \beta^{\beta}}\right]^{(a, b)}}}\left[c x^{c-1}\left(1+x^{c}\right)^{-1}+\alpha \beta x^{\beta-1}\right],
$$

respectively. Graphs of the hazard function are presented in Figure 2. The graphs show different shapes including monotonically decreasing and increasing, bathtub followed by upside-down bathtub and upside-down bathtub shapes. The BLLoGW distribution is flexible to accommodate both monotonic and non-monotonic hazard behaviors that are likely to be encountered when dealing with lifetime and reliability data. 


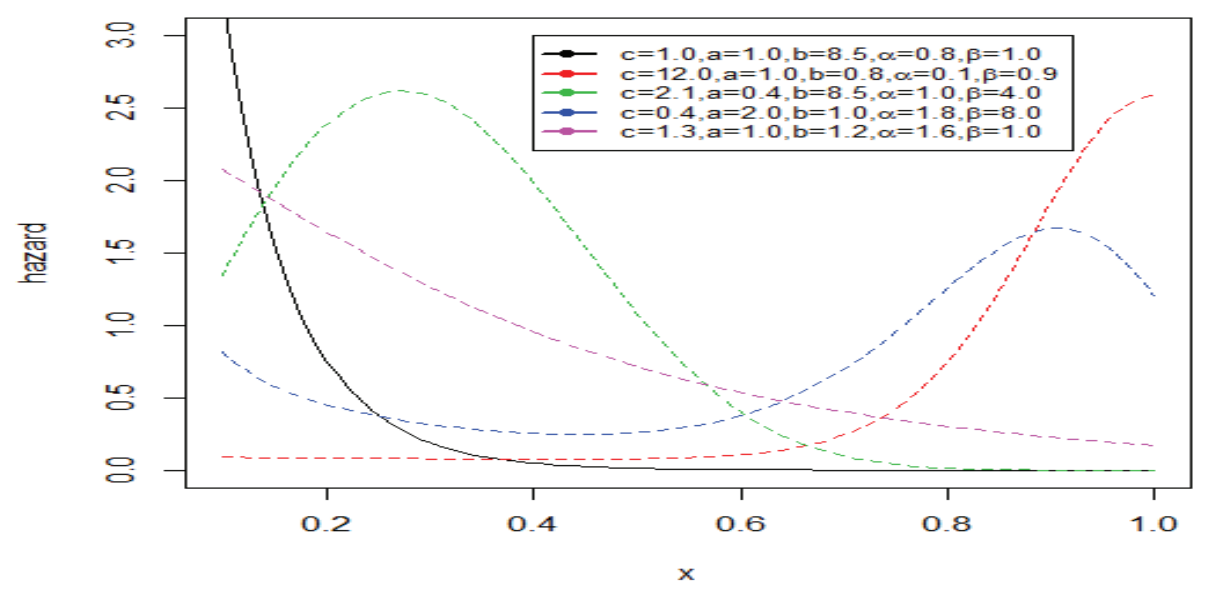

Figure 2. Plots of the BLLoGW Hazard Function

\subsection{Some Sub-models}

In this subsection, the nested-models of the BLLoGW distribution are presented.

- When $\beta=1$, we obtain beta log-logistic exponential (BLLoGE) distribution. The cdf of BLLoGE distribution is given by

$$
F_{B L L O G E}(x ; c, a, b, \alpha)=\frac{1}{B(a, b)} \int_{0}^{G_{L L O G E}(x ; c, \alpha)} t^{a-1}(1-t)^{b-1} d t, \quad c, a, b, \alpha>0 .
$$

- When $\beta=2$, we obtain beta log-logistic Rayleigh (BLLoGR) distribution. The cdf of BLLoGR distribution is given by

$$
F_{B L L O G R}(x ; c, a, b, \alpha)=\frac{1}{B(a, b)} \int_{0}^{G_{L L o G R}(x ; c, \alpha)} t^{a-1}(1-t)^{b-1} d t, \quad c, a, b, \alpha>0 .
$$

- When $\alpha \rightarrow 0^{+}$, we obtain beta log-logistic (BLLoG) distribution. The cdf of BLLoG distribution is given by

$$
F_{B L L o G}(x ; c, a, b)=\frac{1}{B(a, b)} \int_{0}^{G_{L L o G}(x ; c)} t^{a-1}(1-t)^{b-1} d t, \quad c, a, b>0 .
$$

- When $b=1$, the BLLoGW distribution becomes the exponentiated log-logistic Weibull (ELLoGW) distribution. The cdf of ELLoGW distribution is given by

$$
F_{E L L o G W}(x ; c, a, \alpha, \beta)=\left[1-\left(1+x^{c}\right)^{-1} e^{-\alpha x^{\beta}}\right]^{a}, \quad c, a, \alpha, \beta>0 .
$$

- When $b=1$ and $\beta=1$, the BLLoGW distribution becomes the exponentiated log-logistic exponential (ELLoGE) distribution and the cdf is given by

$$
F_{E L L O G E}(x ; c, a, \alpha,)=\left[1-\left(1+x^{c}\right)^{-1} e^{-\alpha x}\right]^{a}, \quad c, a, \alpha>0 .
$$

- When $\alpha \rightarrow 0^{+}$and $b=1$, the BLLoGW distribution becomes the exponentiated log-logistic (ELLoG) distribution. The cdf of ELLoG distribution is given by

$$
F_{E L L O G}(x ; c, a)=\left[1-\left(1+x^{c}\right)^{-1}\right]^{a}, \quad c, a>0 .
$$

- When $a=1$, we obtain Lehmann type-II log-logistic Weibull (LELLoGW) distribution. The cdf of LELLoGW distribution is given by

$$
F_{L E L L o G W}(x ; c, b, \alpha, \beta)=1-\left[\left(1+x^{c}\right)^{-1} e^{-\alpha x^{\beta}}\right]^{b}, \quad c, b, \alpha, \beta>0 .
$$


- When $a=1$ and $\beta=1$, we obtain Lehmann type-II log-logistic exponential (LELLoGE) distribution. The cdf is given by

$$
F_{\text {LELLoGE }}(x ; c, b, \alpha)=1-\left[\left(1+x^{c}\right)^{-1} e^{-\alpha x}\right]^{b}, \quad c, b, \alpha>0 .
$$

- When $a=1$ and $\beta=2$, we obtain Lehmann type-II log-logistic Rayleigh (LELLoGR) distribution. The cdf is given by

$$
F_{L E L L O G R}(x ; c, b, \alpha)=1-\left[\left(1+x^{c}\right)^{-1} e^{-\alpha x^{2}}\right]^{b}, \quad c, b, \alpha>0 .
$$

- When $\alpha \rightarrow 0^{+}$, and a=1, we obtain Lehmann type-II log-logistic (LELLoG) distribution. The cdf is given by

$$
F_{L E L L O G}(x ; c, b,)=1-\left[\left(1+x^{c}\right)^{-1}\right]^{b}, \quad c, a, b>0 .
$$

- When $a=b=1$, the BLLoGW cdf reduces to the log-logistic Weibull (LLoGW) distribution. The cdf is given by

$$
F_{\text {LLoGW }}(x ; c, \alpha, \beta)=1-\left(1+x^{c}\right)^{-1} e^{-\alpha x^{\beta}}, \quad c, \alpha, \beta>0 .
$$

- When $a=b=\beta=1$, the BLLoGW cdf reduces to the log-logistic exponential (LLoGE) cdf and is given by

$$
F_{\text {LLOGE }}(x ; c, \alpha)=1-\left(1+x^{c}\right)^{-1} e^{-\alpha x}, \quad c, \alpha>0 .
$$

- When $a=b=1$ and $\alpha \rightarrow 0^{+}$, the BLLoGW cdf reduces to the log-logistic (LLoG) cdf and is given by

$$
F_{L L O G}(x ; c)=1-\left(1+x^{c}\right)^{-1}, \quad c>0 .
$$

\subsection{Quantile Function}

To obtain the quantile function of the EBW distribution, we can invert the following equation: $F_{B L L O W}(x)=u, 0 \leq u \leq 1$, that is,

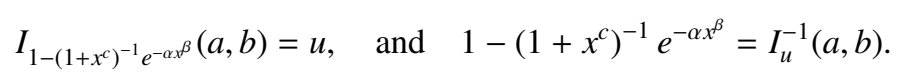

Consequently, we obtain the quantile function of the BLLoGW distribution by solving the equation (25) using numerical methods.

$$
\log \left(1+x^{c}\right)+\alpha x^{\beta}+\log \left(1-I_{u}^{-1}(a, b)\right)=0,
$$

Consequently, random number can be generated based on equation (25). The quantile for selected values of the BLLoGW distribution parameters are listed in Table 1.

Table 1. BLLoGW quantile for selected values

\begin{tabular}{rrrrrr}
\hline & \multicolumn{5}{c}{$(c, a, b, \alpha, \beta)$} \\
\cline { 2 - 6 }$u$ & $(0.8,0.5,5.0,7.0,4.0)$ & $(1.0,3.0,2.5,5.0,1.8)$ & $(1.2,2.0,3.0,3.0,0.6)$ & $(2.2,4.5,1.0,3.5,5.5)$ & $(0.9,10.0,12.0,0.9,4.0)$ \\
\hline 0.1 & 0.0157 & 0.2224 & 0.7348 & 0.1195 & 0.5233 \\
0.2 & 0.0379 & 0.5026 & 0.7865 & 0.1567 & 0.5909 \\
0.3 & 0.0656 & 0.6677 & 0.8205 & 0.1885 & 0.6400 \\
0.4 & 0.1005 & 0.7299 & 0.8473 & 0.2193 & 0.6821 \\
0.5 & 0.1442 & 0.7685 & 0.8707 & 0.2514 & 0.7215 \\
0.6 & 0.2009 & 0.7983 & 0.8924 & 0.2868 & 0.7609 \\
0.7 & 0.2784 & 0.8247 & 0.9139 & 0.3287 & 0.8033 \\
0.8 & 0.3945 & 0.8509 & 0.9373 & 0.3831 & 0.8527 \\
0.9 & 0.6077 & 0.8822 & 0.9667 & 0.4686 & 0.9210 \\
\hline
\end{tabular}




\section{Moments and Conditional Moments}

In this section, we present moments and conditional moments of the BLLoGW distribution.

\section{Moments}

Moments are used to understand different characteristics of a distribution such as skewness, kurtosis, central tendency and dispersion.

Theorem: The $r^{\text {th }}$ non-central moment of the BLLoGW distribution is given by

$$
\begin{aligned}
E\left(X^{r}\right) & =\sum_{i=0}^{\infty} \sum_{m=0}^{\infty} \frac{(-1)^{i+m} \Gamma(a)[\alpha(i+b)]^{m}}{B(a, b) \Gamma(a-i) i ! m !(i+b)}(i+b) B\left(i+b-\frac{r}{c}-\frac{m \beta}{c}, \frac{r+m \beta+c}{c}\right) \\
& +\frac{\alpha(i+b) \beta}{c} B\left(i+b-\frac{r}{c}-\frac{m \beta}{c}-\frac{\beta}{c}, \frac{r+m \beta+\beta}{c}\right) .
\end{aligned}
$$

Proof: Now, we apply the expansion $e^{x}=\sum_{p=0}^{\infty} \frac{x^{p}}{p !}$ to obtain

$$
\begin{aligned}
E\left(X^{r}\right) & =\int_{0}^{\infty} x^{r} f_{\text {BLLoGW }}(x) d x \\
& =\sum_{i=0}^{\infty} \frac{(-1)^{i} \Gamma(a)}{i ! \Gamma(a-i) B(a, b)(i+b)} \int_{0}^{\infty} x^{r}\left(1+x^{c}\right)^{-(i+b)} e^{-\alpha(i+b) x^{\beta}} \\
& \times\left[c x^{c-1}\left(1+x^{c}\right)^{-1}(i+b)+\alpha(i+b) \beta x^{\beta-1}\right] d x \\
& =\sum_{i=0}^{\infty} \sum_{m=0}^{\infty} \frac{(-1)^{i+m} \Gamma(a)[\alpha(i+b)]^{m}}{B(a, b) \Gamma(a-i) i ! m !(i+b)} \int_{0}^{\infty} x^{r+m \beta}\left(1+x^{c}\right)^{-(i+b)} \\
& \times\left[c x^{c-1}\left(1+x^{c}\right)^{-1}(i+b)+\alpha(i+b) \beta x^{\beta-1}\right] d x \\
& =\sum_{i=0}^{\infty} \sum_{m=0}^{\infty} \frac{(-1)^{i+m} \Gamma(a)[\alpha(i+b)]^{m}}{B(a, b) \Gamma(a-i) i ! m !(i+b)}\left[c(i+b) \int_{0}^{\infty} x^{r+m \beta+c-1}\left(1+x^{c}\right)^{-(i+b+1)} d x\right. \\
& \left.+\alpha(i+b) \beta \int_{0}^{\infty} x^{r+m \beta+\beta-1}\left(1+x^{c}\right)^{-(i+b)} d x\right] .
\end{aligned}
$$

Let $y=\left(1+x^{c}\right)^{-1}$, then $x=\left(\frac{1-y}{y}\right)^{\frac{1}{c}}$ and $d x=-\frac{y^{-2}(1-y)^{\frac{1}{c}-1} y^{1-\frac{1}{c}}}{c} d y$, so that

$$
\begin{aligned}
E\left(X^{r}\right) & =\sum_{i=0}^{\infty} \sum_{m=0}^{\infty} \frac{(-1)^{i+m} \Gamma(a)[\alpha(i+b)]^{m}}{B y(a, b) \Gamma(a-i) i ! m !(i+b)}\left[c(i+b) \int_{0}^{1}\left[\left(\frac{1-y}{y}\right)^{\frac{1}{c}}\right]^{r+m \beta+c-1} y^{i+b+1} \frac{y^{-2}(1-y)^{\frac{1}{c}-1} y^{1-\frac{1}{c}}}{c} d y\right. \\
& \left.+\alpha(i+b) \beta \int_{0}^{1}\left[\left(\frac{1-y}{y}\right)^{\frac{1}{c}}\right]^{r+m \beta+\beta-1} y^{i+b} \frac{y^{-2}(1-y)^{\frac{1}{c}-1} y^{1-\frac{1}{c}}}{c} d y\right] \\
& =\sum_{i=0}^{\infty} \sum_{m=0}^{\infty} \frac{(-1)^{i+m} \Gamma(a)[\alpha(i+b)]^{m}}{B(a, b) \Gamma(a-i) i ! m !(i+b)}\left[(i+b) \int_{0}^{1} y^{i+b-\frac{r+m \beta+c}{c}-1}(1-y)^{\frac{r+m \beta+c}{c}-1} d y\right. \\
& +\frac{\alpha(i+b) \beta}{c} \int_{0}^{1} y^{i+b-\frac{r+m \beta+\beta}{c}-1}(1-y)^{\frac{r+m \beta+\beta}{c}-1} d y \\
& =\sum_{i=0}^{\infty} \sum_{m=0}^{\infty} \frac{(-1)^{i+m} \Gamma(a)[\alpha(i+b)]^{m}}{B(a, b) \Gamma(a-i) i ! m !(i+b)}(i+b) B\left(i+b-\frac{r}{c}-\frac{m \beta}{c}, \frac{r+m \beta+c}{c}\right) \\
& +\frac{\alpha(i+b) \beta}{c} B\left(i+b-\frac{r}{c}-\frac{m \beta}{c}-\frac{\beta}{c}, \frac{r+m \beta+\beta}{c}\right),
\end{aligned}
$$

where $B(a, b)=\int_{0}^{1} t^{a-1}(1-t)^{b-1} d t$ is the beta function.

The first six moments, standard deviation (SD), coefficient of variation (CV), coefficient of skewness (CS) and coefficient of kurtosis (CK) for some selected parameters values of the BLLoGW distribution are given in Table 2. The variance $\left(\sigma^{2}\right),(\mathrm{SD}(\sigma)), \mathrm{CV}, \mathrm{CS}$ and $\mathrm{CK}$ are given by

$$
\sigma^{2}=\mu_{2}^{\prime}-\mu^{2}, \quad C V=\frac{\sigma}{\mu}=\frac{\sqrt{\mu_{2}^{\prime}-\mu^{2}}}{\mu}=\sqrt{\frac{\mu_{2}^{\prime}}{\mu^{2}}-1},
$$




$$
C S=\frac{E\left[(X-\mu)^{3}\right]}{\left[E(X-\mu)^{2}\right]^{3 / 2}}=\frac{\mu_{3}^{\prime}-3 \mu \mu_{2}^{\prime}+2 \mu^{3}}{\left(\mu_{2}^{\prime}-\mu^{2}\right)^{3 / 2}}, \quad \text { and } \quad C K=\frac{E\left[(X-\mu)^{4}\right]}{\left[E(X-\mu)^{2}\right]^{2}}=\frac{\mu_{4}^{\prime}-4 \mu \mu_{3}^{\prime}+6 \mu^{2} \mu_{2}^{\prime}-3 \mu^{4}}{\left(\mu_{2}^{\prime}-\mu^{2}\right)^{2}},
$$

respectively.

Table 2. BLLoGW Moments for selected parameter values

\begin{tabular}{|c|c|c|c|c|c|}
\hline \multirow[b]{2}{*}{ Moments } & \multicolumn{5}{|c|}{$(c, a, b, \alpha, \beta)$} \\
\hline & $(8.0,1.0,1.0,2.2,0.9)$ & $(6.5,1.0,1.0,4.5,0.8)$ & $(5.0,1.0,1.0,2.5,0.6)$ & $(4.0,1.0,1.0,2.0,0.9)$ & $(3.0,1.0,1.0,5.0,0.3)$ \\
\hline EX & 0.3774 & 0.1685 & 0.2562 & 0.3992 & 0.0273 \\
\hline EX2 & 0.2507 & 0.0677 & 0.1693 & 0.2839 & 0.0114 \\
\hline EX3 & 0.2094 & 0.0408 & 0.1568 & 0.2686 & 0.0107 \\
\hline EX4 & 0.1989 & 0.0309 & 0.1794 & 0.3130 & 0.0169 \\
\hline EX5 & 0.2070 & 0.0274 & 0.2479 & 0.4393 & 0.0468 \\
\hline EX6 & 0.2331 & 0.0272 & 0.4229 & 0.7419 & 0.2651 \\
\hline $\mathrm{SD}$ & 0.3291 & 0.1982 & 0.3219 & 0.3528 & 0.1029 \\
\hline $\mathrm{CV}$ & 0.8722 & 1.1759 & 1.2562 & 0.8838 & 3.7734 \\
\hline CS & 0.9274 & 2.0684 & 1.8105 & 1.2734 & 8.9839 \\
\hline CK & 3.0807 & 8.1493 & 6.7484 & 5.1151 & 140.6982 \\
\hline
\end{tabular}
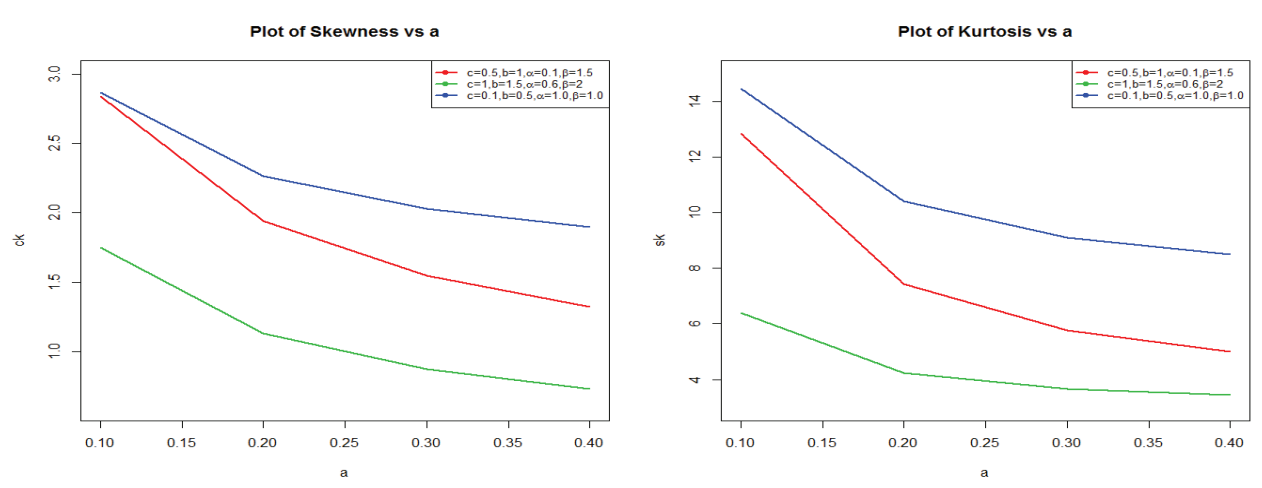

Figure 3. Plot of Skewness and Kurtosis for selected parameter values of the BLLoGW distribution
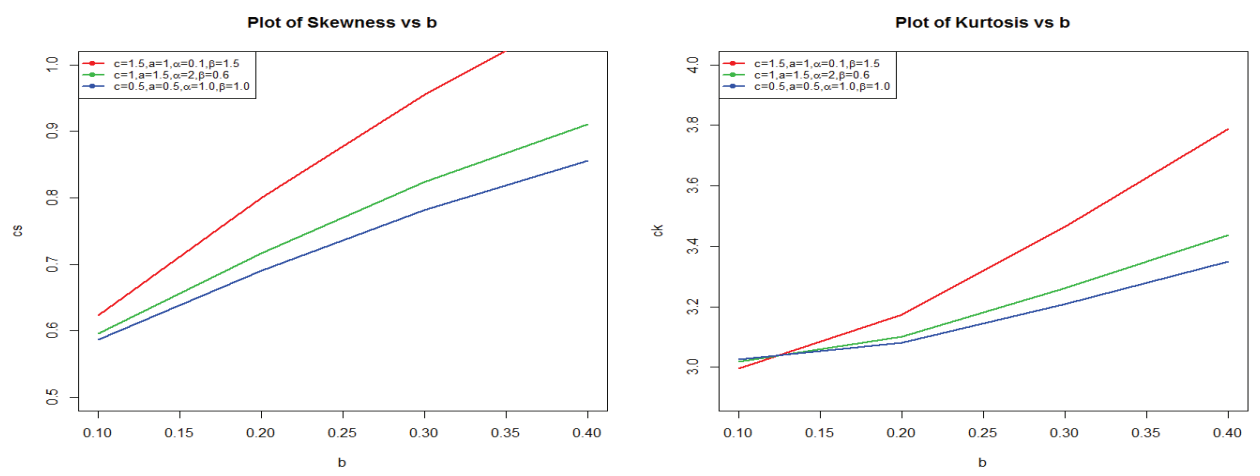

Figure 4. Plot of Skewness and Kurtosis for selected parameter values of the BLLoGW distribution

Plots of skewness and kurtosis of the BLLoGW distribution as a function of different model shape parameters are presented in Figures 3, 4, 5, and 6. The plots shows the dependence of the kurtosis and skewness measures of the shape parameter $a, b, c$ and $\beta$, respectively. Note that plot of the skewness and kurtosis decreases as the parameters $a$ and $\beta$ increase, while the plot of the skewness and kurtosis increase as parameters $b$ and $c$ increase. 

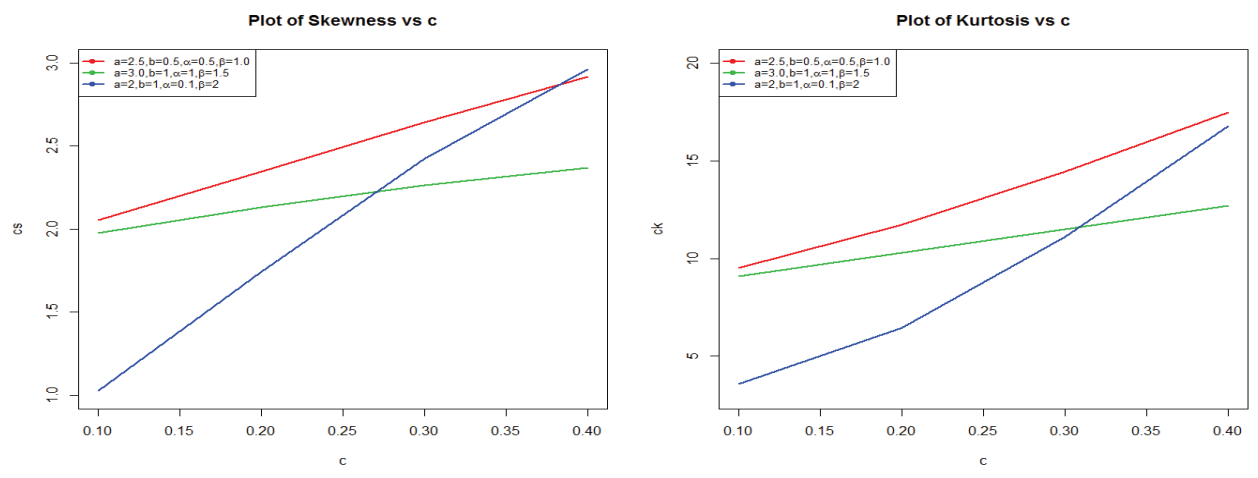

Figure 5. Plot of Skewness and Kurtosis for selected parameter values of the BLLoGW distribution
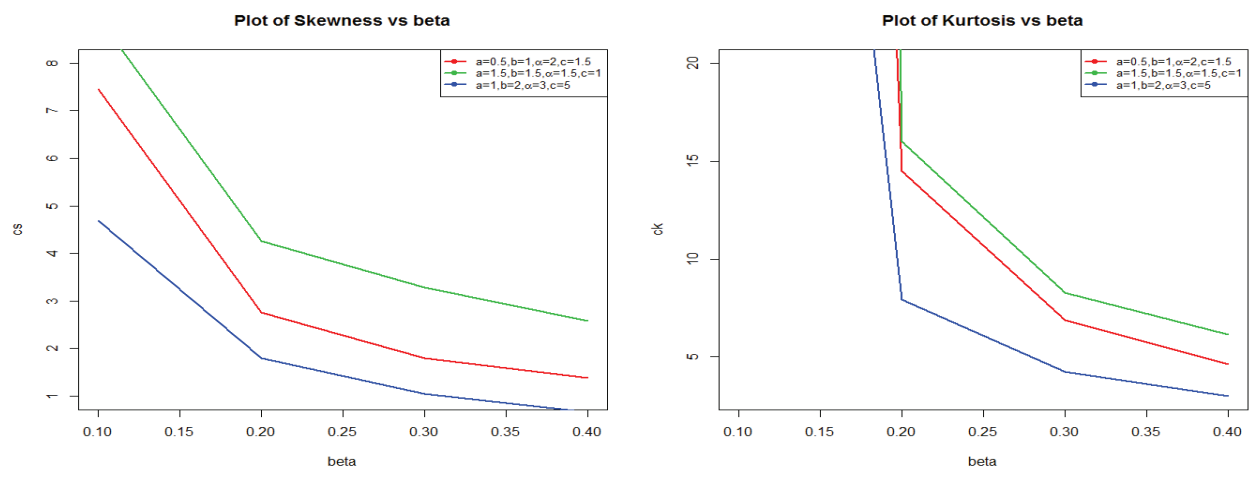

Figure 6. Plot of Skewness and Kurtosis for selected parameter values of the BLLoGW distribution

\subsection{Conditional Moments}

The $r^{\text {th }}$ conditional moment of the BLLoGW distribution is given by

$$
\begin{aligned}
E\left(X^{r} \mid X>t\right) & =\frac{1}{\bar{F}_{B L L o G W}(t)} \int_{t}^{\infty} x^{r} f_{B L L G W}(x) d x \\
& =\frac{1}{\bar{F}_{B L L o G W}(t)} \int_{t}^{\infty} x^{r} \sum_{i=0}^{\infty} \frac{(-1)^{i} \Gamma(a)}{B(a, b)(i+b) i ! \Gamma(a-i)}\left(1+x^{c}\right)^{-(i+b)} e^{-\alpha(i+b) x^{\beta}} \\
& \times\left[c x^{c-1}\left(1+x^{c}\right)^{-1}(i+b)+\alpha(i+b) \beta x^{\beta-1}\right] d x \\
& =\frac{1}{\bar{F}_{B L L o G W}(t)} \sum_{i=0}^{\infty} \sum_{m=0}^{\infty} \frac{(-1)^{i+m}[\alpha(i+b)]^{m} \Gamma(a)}{B(a, b)(i+b) i ! m ! \Gamma(a-i)} \\
& \times(i+b) B_{\left[1+t^{c}\right]^{-1}}\left(i+b-\frac{r}{c}-\frac{m \beta}{c}, \frac{r+m \beta+c}{c}\right) \\
& +\frac{\alpha(i+b) \beta}{c} B_{\left[1+t^{c}\right]^{-1}}\left(i+b-\frac{r}{c}-\frac{m \beta}{c}-\frac{\beta}{c}, \frac{r+m \beta+\beta}{c}\right)
\end{aligned}
$$

where $B_{y}(a, b)=\int_{0}^{y} t^{a-1}(1-t)^{b-1} d t$ is the incomplete beta function. The mean residual life function $E(X-t \mid X>t)$ can be readily obtained.

\section{Mean Deviations, Bonferroni and Lorenz Curves}

The amount of scatter in a population can be measured to some extent by the totality of deviations from the mean and median. These are known as the mean deviation about the mean and the mean deviation about the median.

Bonferroni and Lorenz curves are widely used tool for analyzing and visualizing income inequality. Lorenz curve, $L(p)$ can be regarded as the proportion of total income volume accumulated by those units with income lower than or equal to the volume $x$, and Bonferroni curve, $B(p)$ is the scaled conditional mean curve, that is, ratio of group mean income of the population. Bonferroni and Lorenz curves have applications not only in economics for the study income and poverty, 
but also in other fields such as reliability, demography, insurance and medicine. Mean deviations, Lorenz and Bonferroni curves for the BLLoGW distribution are presented in this section.

\subsection{Mean Deviations}

The mean deviation about the mean $\mu$ and the mean deviation about the median $\mathrm{M}$ are defined by

$$
\delta_{\mu}(X)=\int_{0}^{\infty}|x-\mu| f(x) d x=2 \mu F(x)(\mu)-2 \mu+2 \int_{\mu}^{\infty} x f(x) d x,
$$

and

$$
\delta_{M}(X)=\int_{0}^{\infty}|x-M| f(x) d x=-\mu+2 \int_{M}^{\infty} x f(x) d x .
$$

respectively. The mean deviation about the mean $\mu$ for BLLoGW distribution is given by

$$
\delta_{1}(X)=2 \mu F_{B L L O G W}(\mu)-2 \mu+2 T(\mu),
$$

where

$$
\begin{aligned}
T(\mu) & =\int_{\mu}^{\infty} x f_{B L L o G W}(x) d x \\
& =\sum_{i=0}^{\infty} \sum_{m=0}^{\infty} \frac{(-1)^{i+m}[\alpha(i+b)]^{m} \Gamma(a)}{B(a, b)(i+b) i ! m ! \Gamma(a-i)}(i+b) B_{\left[1+\mu^{c}\right]^{-1}}\left(i+b-\frac{1}{c}-\frac{m \beta}{c}, \frac{1+m \beta+c}{c}\right) \\
& +\frac{\alpha(i+b) \beta}{c} B_{\left[1+\mu^{c}\right]^{-1}}\left(i+b-\frac{1}{c}-\frac{m \beta}{c}-\frac{\beta}{c}, \frac{1+m \beta+\beta}{c}\right) .
\end{aligned}
$$

The mean deviation about the median $\mathrm{M}$ for BLLoGW distribution is given by

$$
\delta_{2}(X)=-\mu+2 T(M),
$$

where

$$
\begin{aligned}
T(M) & =\int_{M}^{\infty} x f_{B L L o G W}(x) d x \\
& =\sum_{i=0}^{\infty} \sum_{m=0}^{\infty} \frac{(-1)^{i+m}[\alpha(i+b)]^{m} \Gamma(a)}{B(a, b)(i+b) i ! m ! \Gamma(a-i)}(i+b) B_{\left[1+M^{c}\right]^{-1}}\left(i+b-\frac{1}{c}-\frac{m \beta}{c}, \frac{1+m \beta+c}{c}\right) \\
& +\frac{\alpha(i+b) \beta}{c} B_{\left[1+M^{c}\right]^{-1}}\left(i+b-\frac{1}{c}-\frac{m \beta}{c}-\frac{\beta}{c}, \frac{1+m \beta+\beta}{c}\right) .
\end{aligned}
$$

\subsection{Bonferroni and Lorenz Curves}

Lorenz and Bonferroni curves for the BLLoGW distribution are presented in this subsection. Bonferroni curve for the BLLoGW distribution is given by

$$
B(p)=\frac{1}{p \mu} \int_{0}^{q} x f_{B L L o G W}(x) d x=\frac{1}{p \mu}[\mu-T(q)],
$$

where

$$
\begin{aligned}
T(q)=\int_{q}^{\infty} x f_{B L L o G W}(x) d x & =\sum_{i=0}^{\infty} \sum_{m=0}^{\infty} \frac{(-1)^{i+m}[\alpha(i+b)]^{m} \Gamma(a)}{B(a, b)(i+b) i ! m ! \Gamma(a-i)} \\
& \times(i+b) B_{\left[1+q^{c}\right]^{-1}}\left(i+b-\frac{1}{c}-\frac{m \beta}{c}, \frac{1+m \beta+c}{c}\right) \\
& +\frac{\alpha(i+b) \beta}{c} B_{\left[1+q^{c}\right]^{-1}}\left(i+b-\frac{1}{c}-\frac{m \beta}{c}-\frac{\beta}{c}, \frac{1+m \beta+\beta}{c}\right)
\end{aligned}
$$

Lorenz curves for the BLLoGW distribution is given by

$$
L(p)=\frac{1}{\mu} \int_{0}^{q} x f_{B L L O G W}(x) d x=\frac{1}{\mu}[\mu-T(q)],
$$


where $T(q)$ is given above.

\section{Rényi Entropy and Order Statistics}

The concept of entropy plays a vital role in information theory. The entropy of a random variable is defined in terms of its probability distribution and can be shown to be a good measure of randomness or uncertainty. Order statistics play an important role in probability and statistics, particularly in reliability and lifetime data analysis. Rényi entropy and distribution of order statistics from the BLLoGW distribution are presented in this section.

\subsection{Rényi Entropy}

Rényi entropy is defined as

$$
I_{R}(v)=\frac{1}{1-v} \log \left(\int_{0}^{\infty}\left[f_{B L L G W}(x ; c, a, b, \alpha, \beta,)\right]^{v} d x\right), v \neq 1, v>0 .
$$

Note that,

$$
\begin{aligned}
f_{\text {BLLoGW }}^{v}(x) & =\frac{1}{B(a, b)^{v}}\left[1-\left(1+x^{c}\right)^{-1} e^{-\alpha x^{\beta}}\right]^{a v-v}\left[\left(1+x^{c}\right)^{-1} e^{-\alpha x^{\beta}}\right]^{b v}\left[c x^{c-1}\left(1+x^{c}\right)^{-1}+\alpha \beta x^{\beta-1}\right]^{v} \\
& =\frac{1}{B(a, b)^{v}} \sum_{i, j=0}^{\infty} \frac{(-1)^{i+j} \Gamma(a v-v+1)[\alpha(b v+i)]^{j}}{\Gamma(a v-v+1-i) i ! j !}\left(1+x^{c}\right)^{-(b v+i)} x^{j \beta}\left[c x^{c-1}\left(1+x^{c}\right)^{-1}+\alpha \beta x^{\beta-1}\right]^{v} .
\end{aligned}
$$

Therefore,

$$
\begin{aligned}
\int_{0}^{\infty} f_{B L L G W}^{v}(x) d x & =\frac{1}{B(a, b)^{v}} \sum_{i, j=0}^{\infty} \frac{(-1)^{i+j} \Gamma(a v-v+1)[\alpha(b v+i)]^{j}}{\Gamma(a v-v+1-i) i ! j !} \\
& \times \int_{0}^{\infty} x^{j \beta}\left(1+x^{c}\right)^{-(b v+i)}\left[c x^{c-1}\left(1+x^{c}\right)^{-1}+\alpha \beta x^{\beta-1}\right]^{v} d x
\end{aligned}
$$

Using the binomial expansion,

$$
\left[c x^{c-1}\left(1+x^{c}\right)^{-1}+\alpha \beta x^{\beta-1}\right]^{v}=\sum_{k=0}^{\infty}\left(\begin{array}{l}
v \\
k
\end{array}\right)\left[c x^{c-1}\left(1+x^{c}\right)^{-1}\right]^{v-k}\left[\alpha \beta x^{\beta-1}\right]^{k},
$$

we have

$$
\begin{aligned}
\int_{0}^{\infty} f_{B L L O W}^{v}(x) d x & =\frac{1}{B(a, b)^{v}} \sum_{i, j, k=0}^{\infty} \frac{(-1)^{i+j} \Gamma(a v-v+1)[\alpha(b v+i)]^{j}}{\Gamma(a v-v+1-i) i ! j !}\left(\begin{array}{l}
v \\
k
\end{array}\right) \\
& \times(\alpha \beta)^{k}(c)^{v-k} \int_{0}^{\infty} x^{j \beta+\beta k+c v-c k-v}\left(1+x^{c}\right)^{-v+k-b v-i} d x
\end{aligned}
$$

Let $y=\left(1+x^{c}\right)^{-1}$, then

$$
\begin{aligned}
\int_{0}^{\infty} f_{B L L G W}^{v}(x) d x & =\frac{1}{B(a, b)^{v}} \sum_{i, j, k=0}^{\infty} \frac{(-1)^{i+j} \Gamma(a v-v+1)[\alpha(b v+i)]^{j}}{\Gamma(a v-v+1-i) i ! j !}\left(\begin{array}{l}
v \\
k
\end{array}\right)(\alpha \beta)^{k}(c)^{v-k-1} \\
& \times \int_{0}^{1} y^{b v-k+i+v-\frac{j \beta-\beta k-c v+c k+v}{c}-\frac{1}{c}-1}(1-y)^{\frac{j \beta+\beta k+c v-c k-v}{c}+\frac{1}{c}-1} d y \\
& =\frac{1}{B(a, b)^{v}} \sum_{i, j, k=0}^{\infty} \frac{(-1)^{i+j} \Gamma(a v-v+1)[\alpha(b v+i)]^{j}}{\Gamma(a v-v+1-i) i ! j !}\left(\begin{array}{l}
v \\
k
\end{array}\right)(\alpha \beta)^{k}(c)^{v-k-1} \\
& \times B\left(b v-k+i+v-\frac{j \beta-\beta k-c v+c k+v}{c}-\frac{1}{c}, \frac{j \beta+\beta k+c v-c k-v}{c}+\frac{1}{c}\right) .
\end{aligned}
$$

As a result, Rényi entropy is given by

$$
\begin{aligned}
I_{R}(v) & =\frac{1}{1-v} \log \left[\frac{1}{B(a, b)^{v}} \sum_{i, j, k=0}^{\infty} \frac{(-1)^{i+j} \Gamma(a v-v+1)[\alpha(b v+i)]^{j}}{\Gamma(a v-v+1-i) i ! j !}\right. \\
& \times\left(\begin{array}{l}
v \\
k
\end{array}\right)(\alpha \beta)^{k}(c)^{v-k-1} B\left(b v-k+i+v-\frac{j \beta-\beta k-c v+c k+v}{c}-\frac{1}{c}, \frac{j \beta+\beta k+c v-c k-v}{c}+\frac{1}{c}\right)
\end{aligned}
$$


for $v>0, v \neq 1$.

\subsection{Order Statistics}

Suppose that $X_{1}, X_{2}, \ldots, X_{n}$ denote continuous independent BLLoGW random variables with common cdf and pdf. The pdf of the $k^{\text {th }}$ order statistic for a random sample of size $n$ from BLLoGW distribution is given by

$$
\begin{aligned}
g_{k: n}(x) & =\frac{n ! f(x)}{(k-1) !(n-k) !}[F(x)]^{k-1}[1-F(x)]^{n-k} \\
& =\frac{n ! f(x)}{(k-1) !(n-k) !} \sum_{p=0}^{n-k}(-1)^{p}\left(\begin{array}{c}
n-k \\
p
\end{array}\right)\left[\frac{1}{B(a, b)} \sum_{j=0}^{\infty} \frac{(-1)^{j} \Gamma(b)\left[1-\left(1+x^{c}\right)^{-1} e^{-\alpha x^{\beta}}\right]^{a+j}}{\Gamma(b-j) j !(a+j)}\right]^{k+p-1} .
\end{aligned}
$$

Note that,

$$
\begin{aligned}
{[F(x)]^{k+p-1} } & =\left[\frac{1}{B(a, b)} \sum_{j=0}^{\infty} \frac{(-1)^{j} \Gamma(b)\left[1-\left(1+x^{c}\right)^{-1} e^{-\alpha x^{\beta}}\right]^{a+j}}{\Gamma(b-j) j !(a+j)}\right]^{k+p-1} \\
& =\frac{(\Gamma(b))^{p+k-1}}{(B(a, b))^{p+k-1}} \sum_{j=0}^{\infty} d_{a+j, p+k-1}\left[1-\left(1+x^{c}\right)^{-1} e^{-\alpha x^{\beta}}\right]^{j}
\end{aligned}
$$

by using the identity $\left(\sum_{j=0}^{\infty} a_{j} x^{j}\right)^{m}=\sum_{j=0}^{\infty} c_{j, m} x^{j}$ for n positive integer (see Gradshteyn and Ryzshik (2000)), where

$$
a_{j}=\frac{(-1)^{j}}{\Gamma(b-j) j !(a+j)},
$$

$d_{a+j, k+p-1}=\left((a+1) c_{0}\right)^{-1} \sum_{l=1}^{a+j}[(n-i+1) l-a-j+l] c_{l} d_{a+j-1, k+p-1}$ and $d_{0}=c_{k+p-1}^{0}$. Now, using the series representation

$$
\left(1-\left(1+x^{c}\right)^{-1} e^{-\alpha x^{\beta}}\right)^{k+p-1}=\sum_{m=0}^{\infty} \frac{(-1)^{m} \Gamma(k+p)}{m ! \Gamma(k+p-m)}\left(1+x^{c}\right)^{-m} e^{-\alpha(m) x^{\beta}},
$$

we have

$$
[F(x)]^{k+p-1}=\frac{(\Gamma(b))^{k+p-1}}{(B(a, b))^{k+p-1}} \sum_{m=0}^{\infty} \frac{(-1)^{m} \Gamma(k+p)}{m ! \Gamma(k+p-m)}\left(1+x^{c}\right)^{-m} e^{-\alpha(m) x^{\beta}} d_{a+j, k+p-1} .
$$

The distribution of the $k^{\text {th }}$ order statistics is given by:

$$
\begin{aligned}
g_{k: n}(x) & =\frac{n !(\Gamma(b))^{k+p-1} \Gamma(a)}{(k-1) !(n-k) !(B(a, b))^{k+p}} \sum_{p=0}^{n-k} \sum_{i, m=0}^{\infty} \frac{(-1)^{p+m+i} \Gamma(k+p)}{(i+b) \Gamma(a-i) \Gamma(p+k-m) m ! i !} \\
& \times\left(\begin{array}{c}
n-k \\
p
\end{array}\right) d_{a+j, k+p-1}\left(1+x^{c}\right)^{-(i+b+m)} e^{-\alpha(i+b+m) x^{\beta}}\left[c x^{c-1}\left(1+x^{c}\right)^{-1}(i+b)+\alpha(i+b) \beta x^{\beta-1}\right] .
\end{aligned}
$$

The $t^{\text {th }}$ moment of the distribution of the $i^{\text {th }}$ order statistics of BLLoGW distribution can be derived from the result of Barakat and Abdelkader (2004), that is,

$$
E\left[X_{i: n}^{t}\right]=t \sum_{p=n+i-1}^{n}(-1)^{p-n+i-1}\left(\begin{array}{l}
p-1 \\
n-i
\end{array}\right)\left(\begin{array}{l}
n \\
p
\end{array}\right) \int_{0}^{\infty} x^{t-1}[1-F(x)]^{p} d x .
$$

Note that

$$
\begin{aligned}
\int_{0}^{\infty} x^{t-1}\left[1-F_{\text {BLLGG }}(x)\right]^{p} d x & =\sum_{l=0}^{p}(-1)^{l}\left(\begin{array}{l}
p \\
l
\end{array}\right) \int_{0}^{\infty} x^{t-1}\left[F_{B L L o G W}(x)\right]^{l} d x \\
& =\sum_{l=0}^{p}(-1)^{l}\left(\begin{array}{l}
p \\
l
\end{array}\right) \int_{0}^{\infty} \frac{x^{t-1}}{(B(a, b))^{l}}\left[\sum_{j=0}^{\infty} \frac{(-1)^{j} \Gamma(b)\left[1-\left(1+x^{c}\right)^{-1} e^{-\alpha x^{\beta}}\right]^{a+j}}{\Gamma(b-j) j !(a+j)}\right]^{l} d x .
\end{aligned}
$$


That is,

$$
\begin{aligned}
{\left[F_{\text {BLLoGW }}(x)\right]^{l} } & =\left[\frac{1}{B(a, b)} \sum_{j=0}^{\infty} \frac{(-1)^{j} \Gamma(b)\left[1-\left(1+x^{c}\right)^{-1} e^{-\alpha x^{\beta}}\right]^{a+j}}{\Gamma(b-j) j !(a+j)}\right]^{l} \\
& =\frac{\Gamma(b)^{l}}{B(a, b)^{l}}\left[1-\left(1+x^{c}\right)^{-1} e^{-\alpha x^{\beta}}\right]^{a l}\left[\sum_{j=0}^{\infty} \frac{(-1)^{j}\left[1-\left(1+x^{c}\right)^{-1} e^{-\alpha x^{\beta}}\right]^{j}}{\Gamma(b-j) j !(a+j)}\right]^{l}
\end{aligned}
$$

Therefore,

$$
\begin{aligned}
\int_{0}^{\infty} x^{t-1}\left[1-F_{\text {BLLOW }}(x)\right]^{p} d x & =\sum_{l=0}^{p} \frac{(-1)^{l}\left(\begin{array}{l}
p \\
l
\end{array}\right) \Gamma(b)^{l}}{[B(a, b)]^{l}} \int_{0}^{\infty} x^{t-1}\left[1-\left(1+x^{c}\right)^{-1} e^{-\alpha x^{\beta}}\right]^{a l} \\
& \times\left[\sum_{j=0}^{\infty} a_{j}\left[1-\left(1+x^{c}\right)^{-1} e^{-\alpha x^{\beta}}\right]^{j}\right]^{l} d x
\end{aligned}
$$

where

$$
a_{j}=\frac{(-1)^{j}}{\Gamma(b-j) j !(a+j)} .
$$

Using the identity $\left(\sum_{k=0}^{\infty} a_{k} x^{k}\right)^{n}=\sum_{k=0}^{\infty} c_{k, n} x^{k}$ for n positive integer (see Gradshteyn and Ryzshik (2000)), we have

$$
\left[\sum_{j=0}^{\infty} a_{j}\left[1-\left(1+x^{c}\right)^{-1} e^{-\alpha x^{\beta}}\right]^{j}\right]^{l}=\sum_{j=0}^{\infty} c_{j, l}\left[1-\left(1+x^{c}\right)^{-1} e^{-\alpha x^{\beta}}\right]^{j},
$$

where $c_{0, l}=a_{0}^{l}$ and $c_{j, l}=\left(j a_{0}\right)^{-1} \sum_{q=1}^{j}(l q-j+q) a_{q} c_{j-q, l}$. Therefore,

$$
\begin{aligned}
\int_{0}^{\infty} x^{t-1}\left[1-F_{\text {BLLoGW }}(x)\right]^{p} d x & =\sum_{l=0}^{p} \sum_{j=0}^{\infty} \frac{(-1)^{l}\left(\begin{array}{l}
p \\
l
\end{array}\right) \Gamma(b)^{l}}{[B(a, b)]^{l}} c_{j, l} \\
& \times \int_{0}^{\infty} x^{t-1}\left[1-\left(1+x^{c}\right)^{-1} e^{-\alpha x^{B}}\right]^{a l+j} d x
\end{aligned}
$$

Note that

$$
\left[1-\left(1+x^{c}\right)^{-1} e^{-\alpha x^{\beta}}\right]^{a l+j}=\sum_{v=0}^{\infty} \frac{(-1)^{v} \Gamma(a l+j+1)}{\Gamma(a l+j+1-v) v !}\left[\left(1+x^{c}\right)^{-1} e^{-\alpha x^{\beta}}\right]^{v} .
$$

Using the series expansion above, we have

$$
\begin{aligned}
& \int_{0}^{\infty} x^{t-1}\left[1-F_{\text {BLLOGW }}(x)\right]^{p} d x=\sum_{l=0}^{p} \sum_{j, v=0}^{\infty} \frac{(-1)^{l+v}\left(\begin{array}{l}
p \\
l
\end{array}\right) \Gamma(b)^{l} \Gamma(a l+j+1)}{B(a, b)^{l} \Gamma(a l+j+1-v) v !} c_{j, l} \int_{0}^{\infty} x^{t-1}\left[\left(1+x^{c}\right)^{-1} e^{-\alpha x^{\beta}}\right]^{v} d x \\
& =\sum_{l=0}^{p} \sum_{j, v, r=0}^{\infty} \frac{(-1)^{l+v+r}\left(\begin{array}{l}
p \\
l
\end{array}\right) \Gamma(b)^{l} \Gamma(a l+j+1)(\alpha)^{r}(v)^{r}}{B(a, b)^{l} \Gamma(a l+j+1-v) v ! r ! c} c_{j, l} \int_{0}^{1} y^{v-\frac{t}{c}-\frac{r \beta}{c}-1}(1-y)^{\frac{t+r \beta}{c}-1} d y \\
& =\sum_{l=0}^{p} \sum_{j, v, r=0}^{\infty} \frac{(-1)^{l+v+r}\left(\begin{array}{l}
p \\
l
\end{array}\right) \Gamma(b)^{l} \Gamma(a l+j+1)(\alpha)^{r}(v)^{r}}{B(a, b)^{l} \Gamma(a l+j+1-v) v ! r ! c} c_{j, l} B\left(v-\frac{t}{c}-\frac{r \beta}{c}, \frac{t+r \beta}{c}\right) .
\end{aligned}
$$

Therefore, the $t^{\text {th }}$ moment of the distribution of the $i^{\text {th }}$ order statistic from BLLoGW distribution is given by

$$
\begin{aligned}
E\left[X_{i: n}^{t}\right] & =t \sum_{p=n+i-1}^{n}(-1)^{p-n+i-1}\left(\begin{array}{l}
p-1 \\
n-i
\end{array}\right)\left(\begin{array}{l}
n \\
p
\end{array}\right) \int_{0}^{\infty} x^{t-1}\left[1-F_{B L L G W}(x)\right]^{p} d x \\
& =t \sum_{p=n+i-1}^{n} \sum_{l=0}^{p} \sum_{j, v, r=0}^{\infty} \frac{(-1)^{p-n+i+l+v+r-1}\left(\begin{array}{l}
p \\
l
\end{array}\right)\left(\begin{array}{l}
p-1 \\
n-i
\end{array}\right)\left(\begin{array}{l}
n \\
p
\end{array}\right) \Gamma(b)^{l} \Gamma(a l+j+1)(\alpha)^{r}(v)^{r}}{B(a, b)^{l} \Gamma(a l+j+1-v) v ! r ! c} c_{j, l} \\
& \times B\left(v-\frac{t}{c}-\frac{r \beta}{c}, \frac{t+r \beta}{c}\right), \quad \text { for } c>t+r \beta
\end{aligned}
$$




\section{Estimation and Inference}

Let $x_{1}, x_{2}, \ldots, x_{n}$ denote a random sample of size $n$ from BLLoGW distribution and $\varphi=(c, a, b, \alpha, \beta)^{T}$ the vector of parameters. The log-likelihood function of the parameter vector can be written as

$$
\begin{aligned}
\ell=\log L & =(a-1) \sum_{i=1}^{n} \ln \left(1-\left(1+x_{i}^{c}\right)^{-1} e^{-\alpha x_{i}^{\beta}}\right)-(b-1) \sum_{i=1}^{n} \ln \left(\left(1+x_{i}^{c}\right)^{-1} e^{-\alpha x_{i}^{\beta}}\right) \\
& -\ln (B(a, b))-\sum_{i=1}^{n} \ln \left(1+x_{i}^{c}\right)-\alpha \sum_{i=1}^{n} x_{i}^{\beta}+\sum_{i=1}^{n} \ln \left(c x_{i}^{c-1}\left(1+x_{i}^{c}\right)^{-1}+\alpha \beta x_{i}^{\beta-1}\right) .
\end{aligned}
$$

The associated score vector is

$$
U_{n}(\varphi)=\left(\frac{\partial \ell}{\partial c}, \frac{\partial \ell}{\partial \alpha}, \frac{\partial \ell}{\partial \beta}, \frac{\partial \ell}{\partial a}, \frac{\partial \ell}{\partial b}\right)^{T}
$$

where the elements are given by

$$
\begin{aligned}
& \frac{\partial \ell}{\partial c}=(a-1) \sum_{i=1}^{n} \frac{\left(1+x_{i}^{c}\right)^{-2} e^{-\alpha x_{i}^{\beta}} x_{i}^{c} \ln \left(x_{i}\right)}{\left(1-\left(1+x_{i}^{c}\right)^{-1} e^{-\alpha x_{i}^{\beta}}\right)}-(b-1) \sum_{i=1}^{n} \frac{\left(1+x_{i}^{c}\right)^{-2} e^{-\alpha x_{i}^{\beta}} x_{i}^{c} \ln \left(x_{i}\right)}{\left(1+x_{i}^{c}\right)^{-1} e^{-\alpha x_{i}^{\beta}}} \\
& -\sum_{i=1}^{n} \frac{x_{i}^{c} \ln \left(x_{i}\right)}{\left(1+x_{i}^{c}\right)}+\sum_{i=1}^{n} \frac{x_{i}^{c-1}+c x_{i}^{c-1} \ln \left(x_{i}\right)+c x_{i}^{c-1} x_{i}^{c} \ln \left(x_{i}\right)}{c x_{i}^{c-1}\left(1+x_{i}^{c}\right)^{-1}+\alpha \beta x_{i}^{\beta-1}} \text {, } \\
& \frac{\partial \ell}{\partial \beta}=(a-1) \sum_{i=1}^{n} \frac{\left(1+x_{i}^{c}\right)^{-1} e^{-\alpha x_{i}^{\beta}} \alpha x_{i}^{\beta} \ln \left(x_{i}\right)}{\left(1-\left(1+x_{i}^{c}\right)^{-1} e^{-\alpha \alpha_{i}^{\beta}}\right)}+(b-1) \sum_{i=1}^{n} \frac{\left(1+x_{i}^{c}\right)^{-1} e^{-\alpha x_{i}^{\beta}} \alpha x_{i}^{\beta} \ln \left(x_{i}\right)}{\left(1+x_{i}^{c}\right)^{-1} e^{-\alpha x_{i}^{\beta}}} \\
& -\alpha \sum_{i=1}^{n} x_{i}^{\beta} \ln \left(x_{i}\right)+\sum_{i=1}^{n} \frac{\alpha x_{i}^{\beta-1}\left(1+\beta \ln \left(x_{i}\right)\right)}{c x_{i}^{c-1}\left(1+x_{i}^{c}\right)^{-1}+\alpha \beta x_{i}^{\beta-1}} \text {, } \\
& \frac{\partial \ell}{\partial \alpha}=(a-1) \sum_{i=1}^{n} \frac{\left(1+x_{i}^{c}\right)^{-1} x_{i}^{\beta} e^{-\alpha x_{i}^{\beta}}}{\left(1-\left(1+x_{i}^{c}\right)^{-1} e^{-\alpha x_{i}^{\beta}}\right)}+(b-1) \sum_{i=1}^{n} \frac{\left(1+x_{i}^{c}\right)^{-1} x_{i}^{\beta} e^{-\alpha x_{i}^{\beta}}}{\left(1+x_{i}^{c}\right)^{-1} e^{-\alpha x_{i}^{\beta}}} \\
& -\sum_{i=1}^{n} x_{i}^{\beta}+\sum_{i=1}^{n} \frac{\beta x_{i}^{\beta-1}}{c x_{i}^{c-1}\left(1+x_{i}^{c}\right)^{-1}+\alpha \beta x_{i}^{\beta-1}} \text {, } \\
& \frac{\partial \ell}{\partial a}=-(\psi(a)-\psi(a+b))+\sum_{i=1}^{n}\left(1-\left(1+x_{i}^{c}\right)^{-1} e^{-\alpha x_{i}^{\beta}}\right),
\end{aligned}
$$

and

$$
\frac{\partial \ell}{\partial b}=-(\psi(b)-\psi(a+b))+\sum_{i=1}^{n}\left(\left(1+x_{i}^{c}\right)^{-1} e^{-\alpha x_{i}^{\beta}}\right) .
$$

respectively. Solving the nonlinear system equations, $U_{n}(\varphi)=\mathbf{0}$ yields the maximum likelihood estimates. These equations can be solved numerically via iterative methods such as Newton-Raphson technique using statistical software.

We maximized the likelihood function using NLmixed in SAS as well as the function nlm in R (R Development Core Team (2011)). These functions were applied and executed for wide range of initial values. This process often results or lead to more than one maximum, however, in these cases, we take the MLEs corresponding to the largest value of the maxima. In a few cases, no maximum was identified for the selected initial values. In these cases, a new initial value was tried in order to obtain a maximum.

The issues of existence and uniqueness of the MLEs are theoretical interest and has been studied by several authors for different distributions including Seregina(2010), Santos Silva and Tenreyros (2010), Zhou (2009), and Xia, Mi, and 
Zhou (2009). At this point we are not able to address the theoretical aspects (existence, uniqueness) of the MLE of the parameters of the BLLoGW distribution.

\subsection{Asymptotic Confidence Interval}

The Fisher information of the BLLoGW distribution is a $5 \times 5$ symmetric matrix given by $\mathbf{I}(\varphi)=\left[\mathbf{I}_{\theta_{i}, \theta_{j}}\right]_{5 \times 5}=E\left(-\frac{\partial^{2} \log L}{\partial \theta_{i} \partial \theta_{j}}\right)$, $i, j=1,2,3,4,5$. The total Fisher information matrix $n \mathbf{I}(\varphi)$ can be approximated by $\mathbf{I}_{n}(\hat{\varphi}) \approx\left[-\left.\frac{\partial^{2} \ell}{\partial \theta_{i} \partial \theta_{j}}\right|_{\varphi=\hat{\varphi}}\right]_{5 X 5}$, for $i, j=$ $1,2,3,4,5$. Let $\hat{\varphi}=(\hat{a}, \hat{b}, \hat{c}, \hat{\alpha}, \hat{\beta})$ be the maximum likelihood estimates of $\varphi=(a, b, c, \alpha, \beta)$. Under the usual regularity conditions and that the parameters are in the interior of the parameter space, but not on the boundary, are met, we have $\sqrt{n}(\hat{\varphi}-\hat{\varphi}) \stackrel{d}{\rightarrow} N_{5}\left(\underline{0}, \mathbf{I}^{-1}(\varphi)\right)$, where $I(\varphi)$ is the expected Fisher information matrix. The multivariate normal distribution with mean vector $\underline{0}$ and covariance matrix $I(\varphi)$ can be used to construct confidence intervals for the BLLoGW distribution parameters. A large sample $100(1-\xi) \%$ two-sided confidence intervals for BLLoGW distribution parameters: $a, b, c, \alpha$ and $\beta$ are given by: $\hat{a} \pm Z_{\xi / 2} \sqrt{I_{a a}^{-1}(\hat{\varphi})}, \quad \hat{b} \pm Z_{\xi / 2} \sqrt{I_{b b}^{-1}(\hat{\varphi})}, \quad \hat{\alpha} \pm Z_{\xi / 2} \sqrt{I_{\alpha \alpha}^{-1}(\hat{\varphi})} \hat{\beta} \pm Z_{\xi / 2} \sqrt{I_{\beta \beta}^{-1}(\hat{\varphi})}$, and $\hat{c} \pm Z_{\xi / 2} \sqrt{I_{c c}^{-1}(\hat{\varphi})}$, where $Z_{\xi / 2}$ is the $1-\frac{\xi}{2}$ quantile of the standard normal distribution.

\subsection{Likelihood Ratio Test}

The likelihood ratio (LR) test can be used to compare the fit of the BLLoGW distribution with its nested-models for a given data set. For example, to test $a=b=c=1$, the $\operatorname{LR}$ statistic is $\omega=2[\ln (L(\hat{c}, \hat{a}, \hat{b}, \hat{\alpha}, \hat{\beta}))-\ln (L(1,1,1, \tilde{\alpha}, \tilde{\beta}))]$, where $\hat{c}, \hat{a}, \hat{b}, \hat{\alpha}$, and $\hat{\beta}$ are the unrestricted estimates, and $\tilde{\alpha}$ and $\tilde{\beta}$ are the restricted estimates. The LR test rejects the null hypothesis if $\omega>\chi_{d}^{2}$, where $\chi_{d}^{2}$ denote the upper $100 d \%$ point of the $\chi^{2}$ distribution with 3 degrees of freedom.

\section{Simulation Study}

The performance and accuracy of MLEs of the BLLoGW distribution parameters is examined by conducting a simulation study in this section.

The simulation study is repeated $N=2000$ times with each sample size $n=35,50,70,100,200,400,800,1600$ and true parameters values $I: a=1, b=1, c=1, \alpha=0.02, \beta=2.2$ and $I I: a=1, b=1, c=0.8, \alpha=0.5, \beta=3$. In this simulation study, we computed three quantities: the mean estimate, root mean square error and average bias. The mean estimate, average bias (Abias) and root mean square error (RMSE) of the MLE $\hat{\varphi}$ for the parameter $\varphi=a, b, c, \alpha, \beta$ are given by Mean $=\frac{\sum_{i=1}^{N} \hat{\varphi}_{i}}{N}, \quad \operatorname{ABias}(\hat{\varphi})=\frac{\sum_{i=1}^{N}\left(\hat{\varphi}_{i}-\varphi\right)}{N}$ and $\quad R M S E=\sqrt{\frac{\sum_{i=1}^{N}\left(\hat{\varphi}_{i}-\varphi\right)^{2}}{N}}$, respectively. The mean MLEs of the BLLoGW distribution parameters along with their respective root mean squared errors (RMSE) and average bias for different sample sizes are listed in Table 3. 
Table 3. Monte Carlo Simulation Results: Mean, Average Bias and RMSE

\begin{tabular}{|c|c|c|c|c|c|c|c|}
\hline \multirow[b]{2}{*}{ Parameter } & \multirow[b]{2}{*}{$\mathbf{n}$} & \multicolumn{3}{|c|}{$\mathbf{I}$} & \multicolumn{2}{|r|}{ II } & \multirow[b]{2}{*}{ RMSE } \\
\hline & & Mean & Average Bias & RMSE & Mean & Average Bias & \\
\hline \multirow[t]{8}{*}{$a$} & 35 & 9.9150 & 8.9150 & 22.6950 & 10.3933 & 9.3933 & 23.4362 \\
\hline & 50 & 8.4664 & 7.4664 & 22.0104 & 7.8071 & 6.8071 & 17.7589 \\
\hline & 70 & 6.3712 & 5.3712 & 17.5209 & 6.7053 & 5.7053 & 14.6949 \\
\hline & 100 & 5.0748 & 4.0748 & 14.4317 & 6.0698 & 5.0698 & 12.3993 \\
\hline & 200 & 2.5801 & 1.5801 & 6.9832 & 4.4065 & 3.4065 & 9.3691 \\
\hline & 400 & 1.9886 & 0.9886 & 5.1553 & 3.0216 & 2.0216 & 6.1737 \\
\hline & 800 & 1.3256 & 0.3256 & 1.7279 & 1.9382 & 0.9381 & 3.7781 \\
\hline & 1600 & 1.1287 & 0.1287 & 0.5203 & 1.4826 & 0.4826 & 2.4887 \\
\hline \multirow[t]{8}{*}{$b$} & 35 & 9.3545 & 8.3545 & 22.6797 & 9.8245 & 8.8245 & 23.3862 \\
\hline & 50 & 8.0962 & 7.0962 & 21.6772 & 7.2515 & 6.2515 & 17.6077 \\
\hline & 70 & 6.1140 & 5.1140 & 17.1819 & 6.1587 & 5.1587 & 14.2823 \\
\hline & 100 & 4.9397 & 3.9397 & 14.1993 & 5.4726 & 4.4726 & 11.8467 \\
\hline & 200 & 2.5441 & 1.5441 & 6.8594 & 3.8884 & 2.8884 & 8.6754 \\
\hline & 400 & 1.9800 & 0.9800 & 5.1388 & 2.6128 & 1.6158 & 5.5992 \\
\hline & 800 & 1.3172 & 0.3172 & 1.7139 & 1.6353 & 0.6353 & 3.3627 \\
\hline & 1600 & 1.1241 & 0.1241 & 0.5129 & 1.2164 & 0.2164 & 2.1924 \\
\hline \multirow[t]{8}{*}{ c } & 35 & 2.1987 & 1.9187 & 2.0646 & 1.9752 & 1.1752 & 2.0525 \\
\hline & 50 & 1.3183 & 0.3183 & 1.6161 & 1.9222 & 1.1222 & 2.0422 \\
\hline & 70 & 1.2193 & 0.2196 & 1.0583 & 1.7558 & 0.9558 & 1.0843 \\
\hline & 100 & 1.2619 & 0.2619 & 0.9383 & 1.6413 & 0.8413 & 1.0019 \\
\hline & 200 & 1.1557 & 0.1557 & 0.6914 & 1.5667 & 0.7667 & 0.8545 \\
\hline & 400 & 1.0669 & 0.0669 & 0.5177 & 1.1015 & 0.3015 & 0.7588 \\
\hline & 800 & 1.0251 & 0.0258 & 0.3651 & 1.0963 & 0.2963 & 0.6174 \\
\hline & 1600 & 1.0067 & 0.0067 & 0.2502 & 1.0517 & 0.2517 & 0.4857 \\
\hline \multirow[t]{8}{*}{$\alpha$} & 35 & 0.7919 & 0.2919 & 1.8947 & 0.7929 & 0.2929 & 1.8015 \\
\hline & 50 & 0.1567 & 0.1367 & 1.0336 & 0.7773 & 0.2773 & 1.7688 \\
\hline & 70 & 0.1248 & 0.1048 & 1.1052 & 0.7159 & 0.2159 & 1.6068 \\
\hline & 100 & 0.0708 & 0.0508 & 0.4115 & 0.7270 & 0.2270 & 1.4274 \\
\hline & 200 & 0.0410 & 0.0210 & 0.1406 & 0.6552 & 0.1552 & 0.9203 \\
\hline & 400 & 0.0275 & 0.0075 & 0.0393 & 0.6354 & 0.1354 & 0.6805 \\
\hline & 800 & 0.0244 & 0.0044 & 0.0243 & 0.5654 & 0.0654 & 0.4141 \\
\hline & 1600 & 0.0221 & 0.0021 & 0.0139 & 0.5429 & 0.0429 & 0.2917 \\
\hline \multirow{8}{*}{$\beta$} & 35 & 3.8670 & 0.8670 & 2.9265 & 3.8255 & 0.8256 & 2.8539 \\
\hline & 50 & 2.6502 & 0.4502 & 1.3907 & 3.6269 & 0.6289 & 2.5258 \\
\hline & 70 & 2.6205 & 0.4205 & 1.2397 & 3.4538 & 0.4538 & 1.9566 \\
\hline & 100 & 2.6051 & 0.4051 & 1.2056 & 3.1443 & 0.1443 & 0.3926 \\
\hline & 200 & 2.5591 & 0.3591 & 0.9352 & 2.9959 & -0.0041 & 0.8291 \\
\hline & 400 & 2.3593 & 0.1593 & 0.6060 & 2.9615 & -0.0385 & 0.6049 \\
\hline & 800 & 2.2581 & 0.0581 & 0.4069 & 2.9874 & -0.0126 & 0.4349 \\
\hline & 1600 & 2.2215 & 0.0215 & 0.2725 & 2.9699 & -0.0301 & 0.3207 \\
\hline
\end{tabular}

From the results in the Table 3, it can be verified that the mean estimates of the parameters converges to the true parameter and the RMSEs decay toward zero as the sample size $n$ increases. We also notice that for all the parametric values, the biases decrease as the sample size $n$ increases.

\section{Application}

In this section, we present an example to illustrate the flexibility of the BLLoGW distribution and its sub-models for data fitting. We compared the fit of the BLLoGW distribution with the fits of the nested BLLoG, ELLoG, LLoG distributions and the non-nested beta Weibull log-logistic (BWLLoG) distribution. The pdf of the BWLLoG distribution (Makubate et al. (2018)) is given by

$$
\begin{aligned}
g_{B W L L O G}(x ; a, b, c, \alpha, \beta) & =\frac{\alpha \beta c}{B(a, b)} x^{c-1}\left(1+x^{c}\right)^{-2} \frac{\left[1-\left(1+x^{c}\right)^{-1}\right]^{\beta-1}}{\left[\left(1+x^{c}\right)^{-1}\right]^{\beta+1}} \\
& \times \exp \left\{-\alpha b\left[\left(1+x^{c}\right)-1\right]^{\beta}\right\}\left[1-\exp \left\{-\alpha\left[\left(1+x^{c}\right)-1\right]^{\beta}\right\}\right]^{a-1}
\end{aligned}
$$

for $a, b, c, \alpha, \beta>0$.

\subsection{Stress-Rupture Life of Kevlar 49/Epoxy Strands Data}

This data reported by Cooray and Ananda (2008) consist of observations that represent the stress-rupture life of kevlar 49/epoxy strands which are subjected to constant sustained pressure at the $90 \%$ stress level until all have failed, so that 
the complete dataset with the exact times of failure is recorded and shown in Table ??. Andrews and Herzberg (1985) and Barlow, Toland and Freeman (1984) gives the recorded failure times in hours. Initial values for BLLoGW model in R code are $a=0.8, b=1.0, c=0.2, \alpha=1.0, \beta=2.0$.

Table 4. Failure times data of kevlar 49/epoxy strands with pressure at $90 \%$

\begin{tabular}{llllllllllllll}
\hline 0.01 & 0.01 & 0.02 & 0.02 & 0.02 & 0.03 & 0.03 & 0.04 & 0.05 & 0.06 & 0.07 & 0.07 & 0.08 & 0.09 \\
0.09 & 0.10 & 0.10 & 0.11 & 0.11 & 0.12 & 0.13 & 0.18 & 0.19 & 0.20 & 0.23 & 0.24 & 0.24 & 0.29 \\
0.34 & 0.35 & 0.36 & 0.38 & 0.40 & 0.42 & 0.43 & 0.52 & 0.54 & 0.56 & 0.60 & 0.60 & 0.63 & 0.65 \\
0.67 & 0.68 & 0.72 & 0.72 & 0.72 & 0.73 & 0.79 & 0.79 & 0.80 & 0.80 & 0.83 & 0.85 & 0.90 & 0.92 \\
0.95 & 0.99 & 1.00 & 1.01 & 1.02 & 1.03 & 1.05 & 1.10 & 1.10 & 1.11 & 1.15 & 1.18 & 1.20 & 1.29 \\
1.31 & 1.33 & 1.34 & 1.40 & 1.43 & 1.45 & 1.50 & 1.51 & 1.52 & 1.53 & 1.54 & 1.54 & 1.55 & 1.58 \\
4.20 & 4.69 & 7.89 & & & & & & & & & & & \\
\hline
\end{tabular}

The asymptotic covariance matrix of the MLE's for the BLLoGW distribution $\mathbf{I}_{n}^{-1}(\hat{\varphi})$ is given by:

$$
\left(\begin{array}{ccccc}
232.886258 & 202.793181 & -4.46670232 & -5.09291312 & -4.69378563 \\
202.793181 & 178.012075 & -3.83400390 & -4.61840501 & -3.87711698 \\
-4.46670232 & -3.83400390 & 0.08883694 & 0.09040585 & 0.09748933 \\
-5.0929132 & -4.61840501 & 0.09040585 & 0.13669012 & -0.07204885 \\
-4.693786 & 3.87711698 & 0.09748933 & -0.07204885 & 0.14308952
\end{array}\right)
$$

and the approximate $95 \%$ two-sided confidence interval for the parameters $a, b, c, \alpha$ and $\beta$ are given by:

$$
6.46809 \pm 29.9107982,5.07658 \pm 26.1505485,0.22372 \pm 0.5841883,0.244637 \pm 0.7246439,
$$

and $0.93981 \pm 0.7414126$, respectively.

Table 5 gives the maximum likelihood estimates (MLE), standard errors (in parentheses), Akaike Information Criterion (AIC), Bayesian Information Criterion (BIC), Consistent Akaike Information Criterion (AICC) and Hannan-Quinn information criterion (HQIC) of BLLoGW, BLLoG, ELLoG, LLoG and BWLLoG distributions. The values of the goodnessof-fit statistics $W^{*}$ and $A^{*}$ are also presented. The smaller the values of these statistics the better the fit. For the probability plot, we plotted $F\left(y_{(j)} ; \hat{c}, \hat{\alpha}, \hat{\beta}, \hat{a}, \hat{b}\right)$ against $\frac{j-0.375}{n+0.25}, j=1,2, \cdots, n$, where $y_{(j)}$ are the ordered values of the observed data. The measures of closeness are given by the sum of squares

$$
S S=\sum_{j=1}^{n}\left[F\left(y_{(j)}\right)-\left(\frac{j-0.375}{n+0.25}\right)\right]^{2} .
$$

\begin{tabular}{|c|c|c|c|c|c|c|c|c|c|c|c|c|}
\hline \multirow[b]{2}{*}{ Model } & \multicolumn{5}{|c|}{ Estimates } & \multicolumn{7}{|c|}{ Statistics } \\
\hline & $\hat{a}$ & $\hat{b}$ & $\hat{c}$ & $\hat{\alpha}$ & $\hat{\beta}$ & $-2 \log L$ & $A I C$ & AICC & $B I C$ & $H I Q C$ & $W^{*}$ & $A^{*}$ \\
\hline \multirow[t]{2}{*}{ BLLoGW } & 6.468097 & 5.07658 & 0.22372 & 0.24463 & 0.93981 & 204.0771 & 214.0771 & 214.7087 & 227.1527 & 219.3705 & 0.1333 & 0.8042 \\
\hline & $(15.26061)$ & (13.34212) & $(0.29806)$ & $(0.36972)$ & $(0.37827)$ & & & & & & & \\
\hline \multirow[t]{2}{*}{ BLLoG } & 0.581650 & 1.091929 & 1.295956 & - & - & 462.1078 & 468.1078 & 468.3552 & 475.9531 & 471.2838 & 0.4176 & 2.2683 \\
\hline & $(0.036581)$ & $(0.133559)$ & $(0.108778)$ & . & - & & & & & & & \\
\hline \multirow[t]{2}{*}{ ELLoG } & 1.211659 & 0.570392 & - & - & - & 587.683 & 591.683 & 3591.8055 & 596.9133 & 593.8004 & 0.4388 & 2.3777 \\
\hline & $(0.096551)$ & $(0.034775)$ & - & - & - & & & & & & & \\
\hline \multirow[t]{2}{*}{ LLoG } & 1.2265 & & - & . & . & 237.589 & 239.589 & 239.6294 & 242.2041 & 240.6476 & 0.4760 & 2.5805 \\
\hline & $(0.1057)$ & . & . & . & - & & & & & & & \\
\hline \multirow[t]{2}{*}{ BWLLOG } & 0.70773 & 0.15311 & 1.45779 & 5.74192 & 0.72356 & 204.8205 & 214.8205 & 215.4521 & 227.8961 & 220.1139 & 0.4673 & 3.3332 \\
\hline & $(0.21792)$ & $(0.15378)$ & (84.60684) & $(6.12430)$ & $(41.99367)$ & & & & & & & \\
\hline
\end{tabular}

Table 5. Estimation of Models for Failure Times of Kevlar Dataset 
The LR statistics for testing the hypothesis $H_{0}$ : BLLoG against $H_{a}$ : BLLoGW, $H_{0}$ : ELLoG against $H_{a}$ : BLLoGW and $H_{0}$ : LLOG against $H_{a}$ : BLLoGW are 258.0299 (p-value < 0.0001), 374.6059 (p-value < 0.0001), 33.5119 (p-value < 0.0001 ), respectively. Since the p-values are small, we reject the null hypothesis and conclude that the BLLoGW distribution is significantly different from BLLoG, ELLoG and LLoG distributions. The BLLoGW distribution is the better model since it has the smallest value for each of the statistics: AIC, AICC, BIC and HIQC. The values of the goodness-of-fit statistics $W^{*}$ and $A^{*}$ gives clear and convincing evidence that the BLLoGW distribution fits better for kevlar 49/epoxy strands data. Plots of the estimated pdf of the BLLoGW, BLLoG, ELLoG, LLoG and BWLLoG distributions fitted to the dataset are given in Figure 7. The Figure showed that BLLoGW distribution is good lifetime distribution when compared with the three sub-models and the non-nested BWLLoG distribution. Also, the Probability Plots of the compared distributions using the failure times of the kevlar data set is shown in Figure 8. The value of SS from the probability plots is smallest for the BLLoGW distribution.

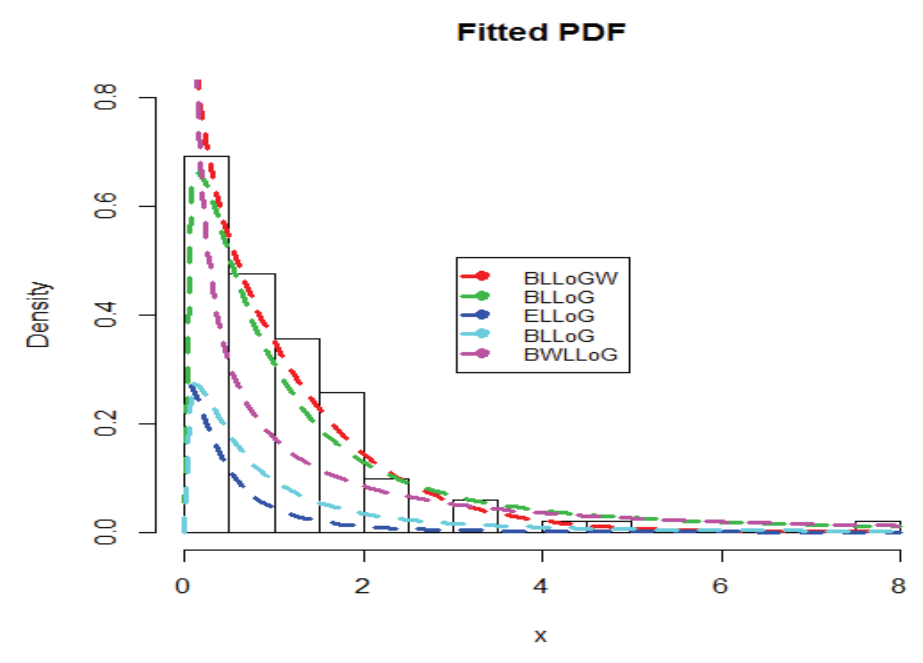

Figure 7. Fitted PDF for Failure Times of Kevlar Data Set

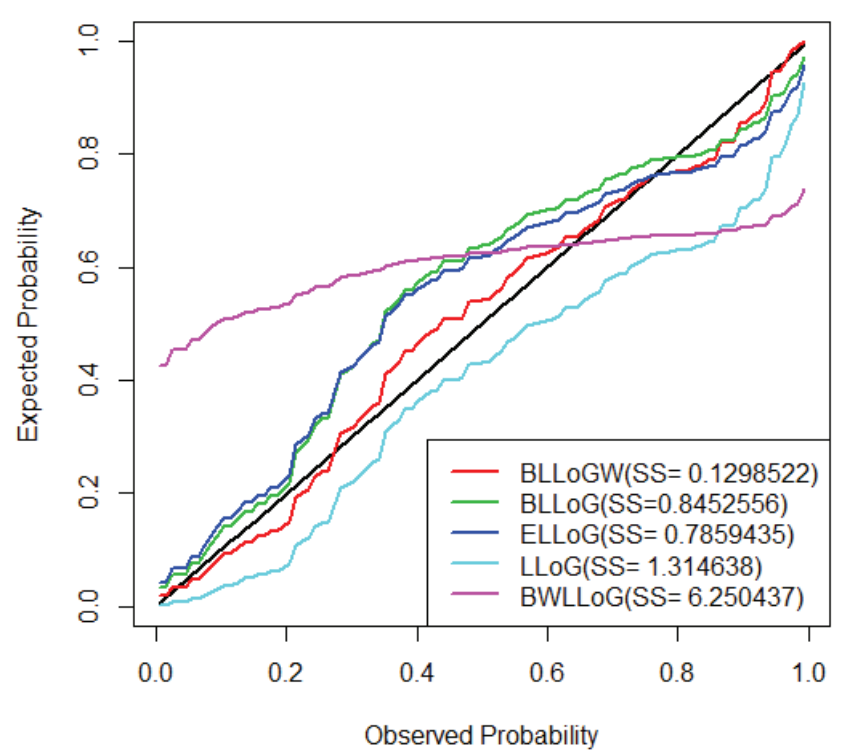

Figure 8. Probability Plots for Failure Times of Kevlar Data Set 


\section{Concluding Remarks}

A new and generalized distribution called the beta log-logistic Weibull (BLLoGW) distribution has been proposed and studied. The BLLoGW distribution has several new and well known distributions as special cases. The BLLoGW distribution possesses hazard function with very flexible behavior. We also obtain closed form expressions for the moments, conditional moments, mean deviations, Bonferroni and Lorenz curves, distribution of order statistics and Rényi entropy. Maximum likelihood estimation technique was used to estimate the model parameters. Finally, the BLLoGW distribution is fitted to a real dataset in order to illustrate its applicability and usefulness.

\section{Disclosure Statement}

The authors declare no conflict of interest.

\section{Acknowledgements}

The authors are very grateful to the editor and referees for reading an earlier version of this manuscript which led to this improved version.

\section{References}

Andrews, D. F., \& Herzberg, A. M. (1985). Data: A Collection of Problems from Many Fields for the Student and Research Worker, Springer Series in Statistics, New York. https://doi.org/10.1007/978-1-4612-5098-2

Barlow, R. E., Toland, R. H., \& Freeman, T. (1984). A Bayesian analysis of stress-rupture life of kevlar 49/epoxy spherical pressure vessels, In: Proceeding of Canadian Conference in Applied Statistics, Marcel Dekker, New York.

Barakat, H., \& Abdelkader, Y. (2004). Computing the moments of order statistics from nonidentical random variables, Stat. Meth. Appl, 13, 15-26. https://doi.org/10.1007/s10260-003-0068-9

Barreto-Souza, W., Santos, A. H. S., \& Cordeiro, G. M. (2010). The beta generalized exponential distribution, Journal of Statistical Computation and Simulation, 80, 159-172.

Cooray, K., \& Ananda, M. (2008). A generalization of the half-Normal distribution with application to lifetime data, Communications in statistics - Theory and Methods, 37, 1323-1337. https://doi.org/10.1080/03610920701826088

Cordeiro, G. M., \& Nadarajah, S. (2011). Closed form expressions for moments of a class of Beta generalized distributions, Brazilian Journal of Probability and Statistics, 25, 14-33. https://doi.org/10.1214/09-BJPS109

Eugene, N., Lee, C., \& Famoye, F. (2002). Beta-normal distribution and its Applications, Communications in Statistics: Theory and Methods, 31, 497-512. https://doi.org/10.1081/STA-120003130

Famoye, F., Lee, C., \& Olumolade, O. (2005). The beta-Weibull Distribution, Journal of Statistical Theory and Applications, 4(2), 121-136.

Gradshteyn, I. S., \& Ryzhik, I. M. (2000). Table of Integrals, Series and Products (sixth edition), San Diego: Academic Press.

Jones, M. C. (2004). Families of distributions arising from distributions of order statistics, Test, 13, 1-43. https://doi.org/10.1007/BF02602999

Lemonte, A. J. (2014). The beta log-logistic distribution, Brazilian Journal of Probability and Statistics, 28, 313-332. https://doi.org/10.1214/12-BJPS209

Makubate, B., Oluyede, B. O., Motobetso, G., Huang, S., \& Fagbamigbe, A. F. (2018). The Beta Weibull-G Family of Distributions : Model, Properties and Application, International Journal of Statistics and Probability, 7(2), 12-32. https://doi.org/10.5539/ijsp.v7n2p12

Nadarajah, S., Cordeiro, G. M., \& Ortega, E. M. M. (2012). General Results for the Beta-Modified Weibull Distribution, Journal of Statistical Computation and Simulations, iFirst, 1-22.

Oluyede, B. O., Foya, S., Warahena-Liyanage, G., \& Huang, S. (2016). The Log-logistic Weibull Distribution with Applications to Lifetime Data, Austrian Journal of Statistics, 45, 43-69. https://doi.org/10.17713/ajs.v45i3.107

Paranaiba, P. F., Ortega, E. M. M., Cordeiro, G. M., \& Pescim, R. R. (2011). The beta Burr XII distribution with application to lifetime data, Computational Statistics and Data Analysis, 55, 1118-1136. https://doi.org/10.1016/j.csda.2010.09.009

R Development Core Team. (2011), A Language and Environment for Statistical Computing, $R$ Foundation for Statistical Computing, Vienna, Austria. 
Santos, S. J. M. C., \& Tenreyro, S. (2010). On the Existence of Maximum Likelihood Estimates in Poisson Regression, Economic Letters, 107, 310-312. https://doi.org/10.1016/j.econlet.2010.02.020

Seregin, A. (2010). Uniqueness of the Maximum Likelihood Estimator for k-Monotone Densities, Proceedings of the American Mathematical Society, 138, 4511-4515. https://doi.org/10.1090/S0002-9939-2010-10496-3

Xia, J., Mi, J., \& Zhou, Y. Y. (2009). On the Existence and Uniqueness of the Maximum Likelihood Estimators of Normal and Log-Normal Population Parameters with Grouped Data, Journal of Probability and Statistics Article id 310575, 16 pages.

Zhou, C. (2009). Existence and Consistency of the Maximum Likelihood Estimator for the Extreme Index, Journal of Multivariate Analysis, 100, 794-815. https://doi.org/10.1016/j.jmva.2008.08.009

\section{Copyrights}

Copyright for this article is retained by the author(s), with first publication rights granted to the journal.

This is an open-access article distributed under the terms and conditions of the Creative Commons Attribution license (http://creativecommons.org/licenses/by/4.0/). 